\title{
Influence of non-radiative exciton recombination in silicon on photoconversion efficiency. 2. Short Shockley-Read-Hall lifetimes
}

\author{
A.V. Sachenko ${ }^{1, *}$, V.P. Kostylyov ${ }^{1}$, V.M. Vlasiuk ${ }^{1}$, R.M. Korkishko ${ }^{1}$, I.O. Sokolovskyi ${ }^{1,2}$, \\ V.V. Chernenko ${ }^{1}$, and M.A. Evstigneev ${ }^{2}$ \\ ${ }^{1} V$. Lashkaryov Institute of Semiconductor Physics, NAS of Ukraine, \\ 41, prospect Nauky, 03028 Kyiv, Ukraine \\ ${ }^{2}$ Department of Physics and Physical Oceanography, \\ Memorial University of Newfoundland, St. John's, NL, A1B $3 X 7$ Canada \\ *E-mail: sach@isp.kiev.ua
}

\begin{abstract}
The influence of non-radiative exciton recombination (NRER) on the photoconversion efficiency in silicon solar cells with short Shockley-Read-Hall lifetimes $\tau_{\mathrm{SRH}}$ has been studied. It has been shown that the efficiency reduction due to this effect is the stronger the shorter $\tau_{\mathrm{SRH}}$. The influence of NRER is most evident when the NRER time becomes shorter than $\tau_{\mathrm{SRH}}$. At sufficiently short $\tau_{\mathrm{SRH}}$, NRER substantially limits the optimal base doping levels of silicon solar cells, at which the photoconversion efficiency is maximal.
\end{abstract}

Keywords: solar cells, photoconversion, non-radiative exciton recombination, ShockleyRead-Hall lifetime.

Manuscript received 15.11.16; revised version received 02.02.17; accepted for publication 01.03.17; published online 05.04.17.

\section{Introduction}

In the first part of this work [1], the influence of nonradiative exciton recombination (NRER) on the photoconversion efficiency in silicon was analyzed for the case of long Shockley-Read-Hall lifetimes $\tau_{\mathrm{SRH}}>100 \mu \mathrm{s}$. It was shown that upon reduction of $\tau_{\mathrm{SRH}}$ the role of this recombination channel increases, which leads to stronger lowering the photoconversion efficiency. Therefore, it is interesting to investigate this effect in the opposite case $\tau_{\mathrm{SRH}}<100 \mu \mathrm{s}$. The respective analysis has been performed in this work.

In contrast to [1], the following criteria are fulfilled: (1) excess electron-hole pair density $\Delta n$ is much lower than the equilibrium density of charge carriers $n_{0}$ in the base region; (2) diffusion length $L_{d}$ of electron-hole pairs is much smaller than the base thickness $d$.

Experimental part of this work includes the illuminated current-voltage curves and photocurrent quantum yield results measured using two $p$ - $n$ junctionbased silicon solar cells (SC) with short values of $\tau_{\mathrm{SRH}}$. Apart from determination of the main characteristics of the SCs studied under AM1.5 conditions, temperature dependence of the short-circuit current $I_{S C}$, open-circuit voltage $V_{O C}$, current-voltage curve fill factor $F F$, and photoconversion efficiency $\eta$ were measured. As our analysis has shown, at very low base doping levels, NRER has practically no effect on these parameters and on their sensitivity to temperature. At the same time, at 
moderate base doping levels this recombination mechanism leads not only to the decrease of these characteristics, but also to a small change in the slope of the curves $V_{O C}(T), F F(T)$, and $\eta(T)$. In the case of small Shockley-Read-Hall lifetimes and heavy base doping, the reduction of photoconversion efficiency caused by the NRER becomes quite essential. For the SC parameters used in this work, it can exceed $40 \%$ of the efficiency value obtained without taking NRER mechanism into account.

\section{Experimental procedure}

SC samples with the structure $p^{+}-n-n^{+}$and $n^{+}-p-p^{+}$were based on $n$ - and $p$-type silicon wafers fabricated using Czochralski method. Because the effect of non-radiative exciton Auger recombination via the deep levels on the photoelectric SC parameters is the stronger the shorter Shockley-Read-Hall lifetime [1], the original Si wavers were chosen with $\tau_{\mathrm{SRH}}$ of about $10^{-6} \ldots 10^{-5} \mathrm{~s}$. The dividing frontal $p^{+}-n$ and isotypic rear $p-p^{+}$junctions were formed by diffusing boron into the phosphorusdoped wafers, and the $n^{+}-p$ and $n-n^{+}$junctions were produced by diffusion of phosphorus into boron-doped wafers. As an antireflecting coating, thermal silicon dioxide layers with the thickness ca. $110 \mathrm{~nm}$ were used.

The initial parameters of silicon and the main properties of the samples studied under AM1.5 conditions are indicated in Table. Here, $n_{0}$ is the doping density, $d-$ sample thickness, $S_{0}-$ surface recombination velocity, and $R_{S}-$ series resistance.

The obtained in this manner SC samples were characterized by very short Shockley-Read-Hall lifetimes, and quite large $p-n$ junction depth, which rendered the photoconversion efficiency below $10 \%$. They were produced using the technology, which allows one to reduce the original lifetime in the base region in order to be able to determine the diffusion length of minority carriers from the open-circuit voltage.

To imitate solar radiation, tungsten lamps with the radiation temperature of $2800 \mathrm{~K}$ were used. The illuminated $I-V$ curves for two SC samples, one with the $n$-type and the other with the $p$-type base, are shown in Fig. 1. The spectral dependence of the internal quantum yield is presented in Fig. 2. The temperature dependence of the short-circuit current, $J_{S C}$, open-circuit voltage, $V_{O C}$, the fill factor, $F F$, and photoconversion efficiency, $\eta$, measured in the range from 25 to $60^{\circ} \mathrm{C}$, are shown in Figs. 3-6.

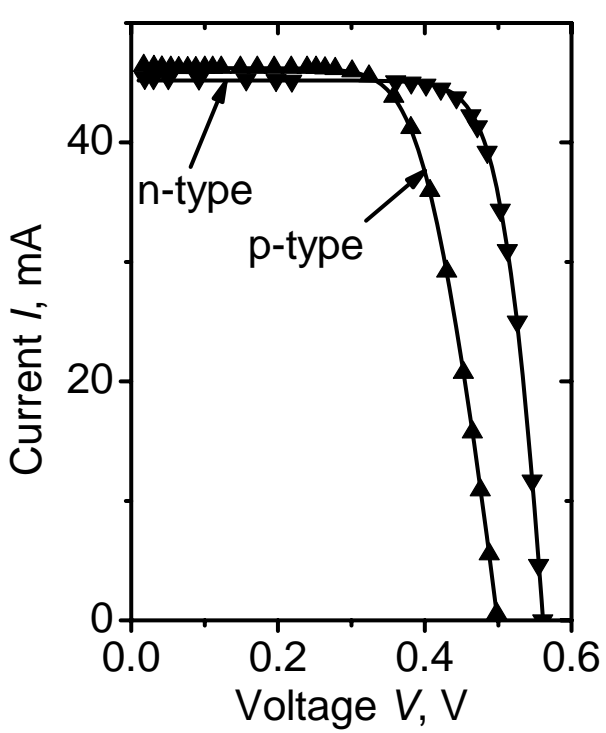

Fig. 1. Theoretical (lines) and experimental (symbols) illuminated current-voltage curves obtained on the SCs with $n$ - and $p$-type base region.

\section{Theoretical analysis}

In our theoretical analysis, the following assumptions were employed to develop a relatively simple analytical description of the experiment.

First, apart from the criterion $L_{d}<d$ we assume that the heavily doped region of the $p-n$ junction has a little effect on the photocurrent internal quantum yield $q(\lambda, T)$. This allows us to use the following expression for this parameter:

$$
q(\lambda, T)=\frac{\alpha(\lambda, T) L_{d}(T)}{1+\alpha(\lambda, T) L_{d}(T)} \exp \left(-\alpha(\lambda, T) d_{p}\right),
$$

where $\alpha(\lambda, T)$ is the absorption coefficient for light of the wavelength $\lambda$ at temperature $T$, and $d_{p}$ - "dead layer" thickness, i.e. the thickness of the heavily doped region.

In general, the short-circuit current density can be written as

$J_{S C}(T)=q\left(1-r_{L}\right) \int_{\lambda_{0}}^{\lambda_{m}(T)} q(\lambda, T) I(\lambda) d \lambda$,

where $q$ is the elementary charge, $r_{L}$ - mean coefficient of photocurrent losses related with the incomplete absorption of light caused by reflection and availability

Table. Silicon parameters and the main characteristics of the samples investigated.

\begin{tabular}{|c|c|c|c|c|c|c|c|c|c|c|}
\hline $\begin{array}{c}\text { Base } \\
\text { type }\end{array}$ & $\begin{array}{c}n_{0}, \\
\mathrm{~cm}^{-3}\end{array}$ & $\begin{array}{c}\tau_{\mathrm{SRH}}, \\
\mathrm{s}\end{array}$ & $A_{S C}, \mathrm{~cm}^{2}$ & $\begin{array}{c}d, \\
\mu \mathrm{m}\end{array}$ & $R_{S}, \mathrm{Ohm}$ & $\begin{array}{c}S_{0}, \\
\mathrm{~cm} / \mathrm{s}\end{array}$ & $J_{S C}, \mathrm{~mA} / \mathrm{cm}^{2}$ & $V_{O C}, \mathrm{~V}$ & $F F, \%$ & $\eta, \%$ \\
\hline$n$ & $3.1 \cdot 10^{15}$ & $2.5 \cdot 10^{-5}$ & 2 & 380 & 0.6 & $2 \cdot 10^{3}$ & 22.4 & 0.568 & 76.6 & 8.31 \\
\hline$p$ & $3 \cdot 10^{15}$ & $7 \cdot 10^{-7}$ & 2 & 350 & 1.5 & $2.25 \cdot 10^{4}$ & 23.1 & 0.503 & 67.5 & 7.86 \\
\hline
\end{tabular}


of the contact grid on the frontal surface of SC, $\lambda_{m}(T)=h c / E_{g}(T)=1.24 \mu \mathrm{m} \cdot \mathrm{eV} / E_{g}(T)$ - absorption threshold wavelength, $E_{g}(T)$ - bandgap value, $\lambda_{0}-$ shortest wavelength that can be absorbed by SC, $I(\lambda)-$ spectral photogeneration intensity of the electron-hole pairs, which depends on the irradiation conditions.

For maximal power conditions of the SCs considered here, the recombination velocity in the spacecharge region (SCR) $S_{S C R}$ does not exceed $30 \mathrm{~cm} / \mathrm{s}$. It is much smaller than the bulk recombination velocity, $S_{d}$, and the front surface recombination velocity, $S_{0}$. Therefore, in the generation-recombination balance equation under the open-circuit conditions, and in the expression for the current-voltage curve, the term related to $S_{S C R}$ can be neglected. Then, the generationrecombination balance equation can be written as

$$
J_{S C}=q\left(S_{d}+S_{0}\right) \Delta n,
$$

where $S_{d}=D / L_{d}, D$ is the minority carriers' diffusion coefficient,

$$
L_{d}=\left[D\left(\frac{1}{\tau_{\mathrm{SRH}}}+\frac{1}{\tau_{r}}+\frac{1}{\tau_{n r}}+\frac{1}{\tau_{\text {Auger }}}\right)^{-1}\right]^{1 / 2}
$$

is the diffusion length, $\tau_{r}=\left(A n_{0}\right)^{-1}-$ radiative recombination time, $\tau_{n r}=\tau_{\mathrm{SRH}}\left(n_{x} / n_{0}\right)$ - NRER time with the parameter $n_{x}=8.2 \cdot 10^{15} \mathrm{~cm}^{-3}$ found in [1], $A=$ $6.3 \cdot 10^{-15} \mathrm{~cm}^{3} / \mathrm{s}$ - radiative recombination parameter [1], $\tau_{\text {Auger }}-$ band-to-band Auger recombination time in $\mathrm{Si}$ determined by Eqs. (18) and (19) of Ref. [2].

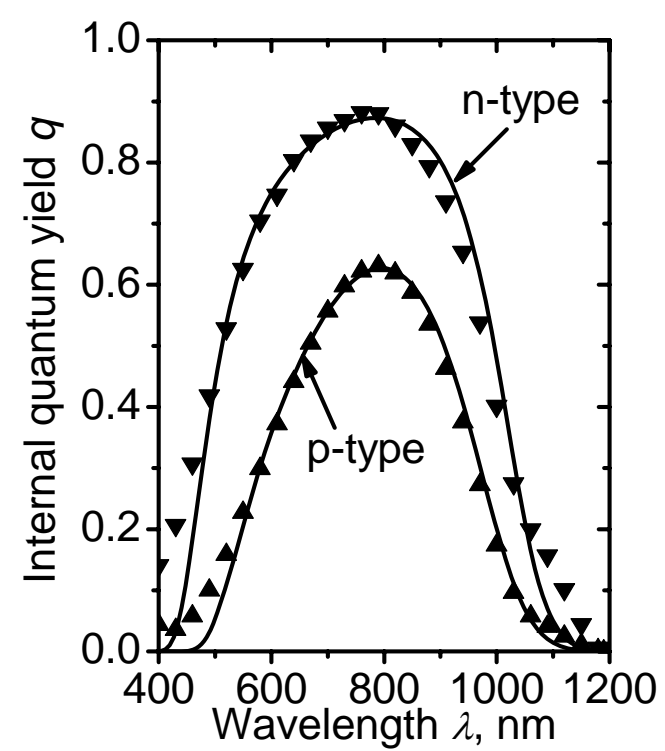

Fig. 2. Theoretical (lines) and experimental (symbols) spectral dependences of the internal quantum yield in SCs.
The illuminated current-voltage curve with the assumptions formulated above, and with the series resistance $R_{S}$ taken into account, has the form:

$$
\begin{aligned}
& I(V)=I_{S C}-q A_{S C}\left(S_{d}+S_{0}\right) \times \\
& \times \frac{n_{i}^{2}(T)}{n_{0}}\left[\exp \left(\frac{q\left(V-I(V) R_{S}\right)}{k T}\right)-1\right],
\end{aligned}
$$

where $n_{i}(T)$ is the intrinsic charge carrier density and $A_{S C}$ is $\mathrm{SC}$ area.

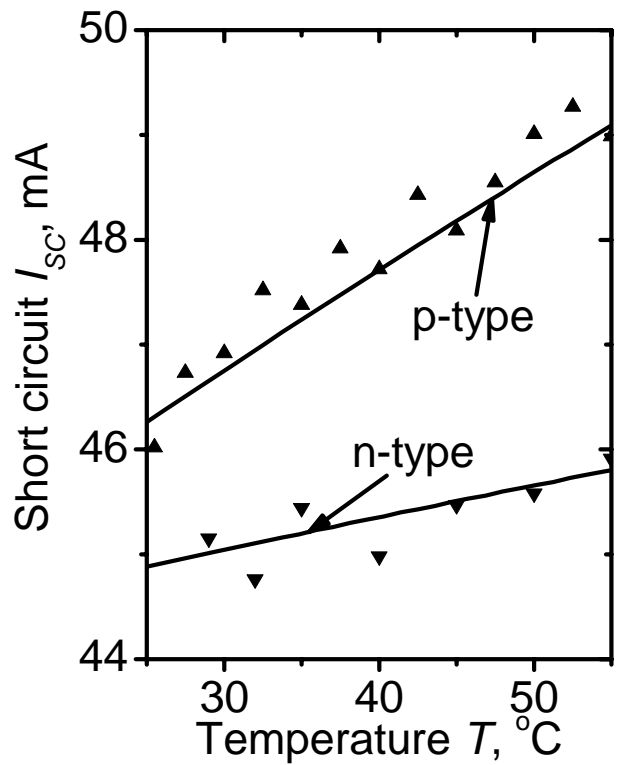

Fig. 3. Theoretical (lines) and experimental (symbols) temperature dependences of the short-circuit current.

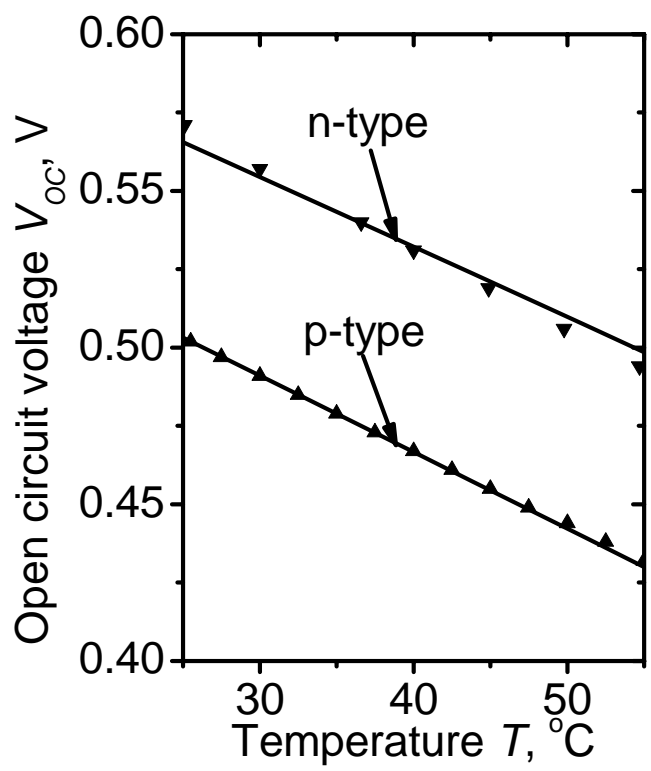

Fig. 4. Theoretical (lines) and experimental (symbols) temperature dependences of the open-circuit voltage. 


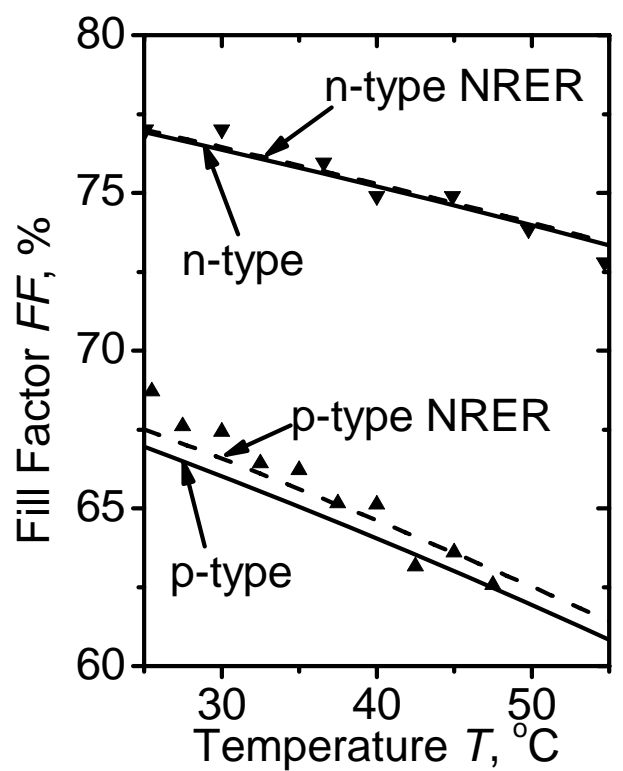

Fig. 5. Theoretical (lines) and experimental (symbols) temperature dependences of the fill factor.

From the maximum-power condition $d(V J(V)) / d V=0$, one can find $V_{m}$, substitution of which into (5) allows one to determine $J_{m}$. As a result, we obtain the usual expression for the photoconversion efficiency

$\eta=\frac{I_{m} V_{m}}{A_{S C} P_{S}}$,

where $P_{S}$ is the incident irradiation power density.

The fill factor is

$F F=\frac{I_{m} V_{m}}{I_{S C} V_{O C}}$,

where the open-circuit voltage is given by the following expression

$V_{O C}=\frac{k T}{q} \ln \left(\frac{J_{S C}}{q\left(S_{d}+S_{0}\right) \frac{n_{i}^{2}(T)}{n_{0}}}+1\right)$.

\section{Experimental results and discussion}

As seen from Fig. 1, the theoretical $I-V$ curves (for the illuminated SC) obtained using Eq. (5) and the parameters from Table agree well with the experimental data.

Fig. 2 shows the experimental and theoretical spectral dependences of the internal quantum yield for two investigated samples. As seen from this figure, the theoretical curves plotted using Eq. (1) agree well with the experiment. For the sample with the $n$-type base, the agreement was obtained for the "dead layer" thickness $d_{p}=0.7 \mu \mathrm{m}$, and for the sample with the $p$-type base, we took $d_{p}=2.5 \mu \mathrm{m}$.
Our analysis has shown that the sensitivity of the short circuit current to the temperature of the environment depends on the irradiation temperature. The lower $T_{\text {eff }}$, the steeper $J_{S C}(T)$ curve. The experimental $J_{S C}(T)$ curves can be described theoretically, if one approximates the imitator spectrum with the Planck spectrum of temperature $T_{\text {eff. }}$ It should be noted that the black-body spectrum is always realized in the longwavelength region near the temperature-dependent absorption edge wavelength $\lambda_{m}(T)=h c / E_{g}(T)$.

If the irradiation source can be modeled as a black (or grey) body, then

$$
J_{S C}(T)=q\left(1-r_{L}\right) B_{0} \int_{\lambda_{0}}^{\lambda_{m}(T)} \frac{q(\lambda, T) d \lambda}{\lambda^{4}\left(\exp \left(\frac{h c}{k T_{e f f} \lambda}\right)-1\right)},
$$

where $B_{0}$ is a constant. In particular, in the case of solar spectrum, $B_{0}=2 \pi c\left(R_{S} / D_{S}\right)^{2} \quad$ [3], where $R_{S}$ is the radius of the Sun, $D_{S}$ - distance from the Sun to the Earth, $h$ - Planck's constant, $c-$ speed of light, and $k-$ Boltzmann's constant.

The luminosity of our solar imitator was chosen so as to obtain the same photocurrent as under AM1.5 conditions:

$$
\begin{aligned}
& 2 \pi c\left(\frac{r_{s}}{D_{s}}\right)^{2 \lambda_{m}(298 K)} \int_{\lambda_{0}}^{\lambda_{m}(298 K)} I_{A M 1.5} q(\lambda, 298 K) d \lambda= \\
& =f\left(R_{L}\right) \int_{\lambda_{0}}^{\lambda^{4}\left(\exp \left(\frac{h c}{k T_{\text {eff }} \lambda}\right)-1\right)},
\end{aligned}
$$

where $I_{\mathrm{AM} 1.5}$ is the solar spectrum under AM1.5 conditions and $f\left(R_{L}\right)$ is the function describing illumination of the sample at the distance $R_{L}$ to the irradiation source.

To model the theoretical $J_{S C}(T)$ curves, we need to take into account the temperature dependences of $\alpha(\lambda, T), E_{g}(T), n_{i}(T), D(T), \tau_{\mathrm{SRH}}(T), \tau_{\text {Auger }}(T)$ and $S_{0}(T)$. The absorption coefficient temperature dependence inherent to $\mathrm{Si}$ was thoroughly investigated in the work [4]. Near the absorption edge, it can be approximated as

$$
\alpha(\lambda, T)=\alpha(\lambda, 298)(T / 298)^{r} \text {. }
$$

The temperature dependence of the bandgap in silicon is well known (see, e.g., [5]). In view of Einstein's relations and due to the fact that at and above room temperature the mobility is determined by phonon scattering and decays with temperature according to the power law $T^{-m}$, the temperature dependence of the diffusion coefficient can be well approximated by an expression $\quad D(T)=D(298 \mathrm{~K}) \cdot(T /(298 \mathrm{~K}))^{-m+1}$. The expression for the Shockley-Read-Hall lifetime in an $n$-type semiconductor has the form 
$\tau_{\mathrm{SRH}} \equiv \frac{C_{p}(T)\left(p_{0}+n_{i}(T) \exp \left(E_{i} / k T\right)\right)+C_{n}(T)\left(n_{0}+n_{i}(T) \exp \left(-E_{i} / k T\right)\right)}{C_{p}(T) C_{n}(T) N_{t} n_{0}}$,

where $p_{0}$ is the equilibrium hole density, $C_{n, p}(T)=V_{n, p} \sigma_{n, p}(T)$ are the electron and hole capture coefficients by a recombination center, $E_{i}$ and $N_{t}$ - energy and concentration of the deep levels, and $V_{n, p}-$ thermal velocities of electrons and holes. As shown in [6], the typical temperature dependence of the capture cross section for holes by recombination centers varies as $\sigma_{p}(T) \sim T^{-k}$ with $k=2$, if the centers are neutral and $k=1 \ldots 3$, if they are negatively charged. Therefore, for low excitation levels, taking the temperature dependence of the thermal velocity into account, for $\sigma_{p}(T) \propto T^{-2}$ we obtain $\tau_{\mathrm{SRH}} \propto T^{3 / 2}$ [7]. If hole capture is due to positively charged centers, $\sigma_{p}(T) \propto \exp \left(-\left(T_{0} / T_{0} / T\right)^{1 / 3}\right) \quad[6]$ and $\tau_{\mathrm{SRH}}(T)$ decreases with temperature.

Because for our SCs, the inequality $\tau_{\mathrm{SRH}} \leq \tau_{\text {Auger }}$ holds, the dependence $L_{d}(T)$ in the case of neutral or attractive recombination centers is given by the relation

$$
\begin{aligned}
& L_{d}(T)=\sqrt{D(T) \tau_{\mathrm{SRH}}(T)}= \\
& =L_{d}(298 \mathrm{~K}) \cdot(T / 298 \mathrm{~K})^{x}, \quad x=\frac{1-m}{2}+\frac{2 k-1}{4},
\end{aligned}
$$

where the parameter $k$ varies between 1 and 3 depending on the exponent of the temperature-dependent capture cross section for the minority carriers.

Note that, generally speaking, the analysis of $\tau_{\mathrm{SRH}}(T)$ should take into account the shift of the Fermi energy towards the middle of the bandgap as the temperature increases. This effect leads to a nonlinearity of $\tau_{\mathrm{SRH}}(T)$, and thus to the non-linearity of $I_{S C}(T)$. In a sufficiently narrow temperature range from 25 to $60{ }^{\circ} \mathrm{C}$ used in our measurements, the nonlinearity of the short-circuit current vs. temperature was not observed. As our estimates show, this is possible, if the recombination level is sufficiently close to the middle of the bandgap.

Fig. 3 shows the experimental short-circuit current vs. temperature curves. The theoretical $I_{S C}(T)$ curves were obtained from the relations (9), (11), and (13) by adjusting the parameter $r+x$, where $r$ and $x$ are the exponents in Eqs. (11) and (13). In the theoretical curves, the sum of these two exponents $r+x \geq 1$; it mainly defines the slope of the $I_{S C}(T)$ curves. As seen from this figure, the agreement between theory and experiment is rather good.

Shown in Figs. 4, 5, and 6 are the experimental temperature curves of $V_{O C}, F F$, and $\eta$ of our SCs. The theoretical counterparts were obtained using the above-introduced formulas with the parameter $n_{x}=$
$8.2 \cdot 10^{15} \mathrm{~cm}^{-3}$. The expressions for $J_{S C}, V_{O C}, F F$, and $\eta$ in the absence of NRER can be obtained as a limiting case of infinite $n_{x}$.

When fitting the experimental results for $V_{O C}, F F$, and $\eta$ under AM1.5 conditions, the fit parameters were $S_{0}$ and $R_{S}$. Their values that allow one to obtain good agreement of the theory with experiment are given in Table. Then, determination of the temperature dependence of $V_{O C}, F F$, and $\eta$ was performed taking into account the temperature dependence of $J_{S C}(T)$ (see Fig. 3 and the discussion above), as well as the temperature dependence of $n_{i}(T)$, which plays a crucial role.

As seen from Figs. 4 to 6, the theoretical curves obtained by taking into account NRER agree well with the experimental curves within the temperature range studied. Also, the theoretical expressions for the photoconversion efficiency obtained with and without taking this recombination process into account differ by as much as $3.8 \%$ for $n$-type and $5.1 \%$ for $p$-type samples. Likewise, the slopes of the efficiency vs. temperature curves obtained with and without NRER somewhat differ.

Fig. 7 shows the experimental and theoretical temperature dependence of the photoconversion efficiency temperature coefficient defined as

$\beta(T)=\frac{\eta\left(T_{0}\right)-\eta(T)}{\eta\left(T_{0}\right)\left(T-T_{0}\right)} 100 \%$,

where $T_{0}$ is the initial temperature. As seen in this figure, the magnitude of $\beta(T) \geq 0.5 \% /{ }^{\circ} \mathrm{C}$ is typical for silicon SCs with graded $p-n$ junctions at relatively low photoconversion efficiency [7].

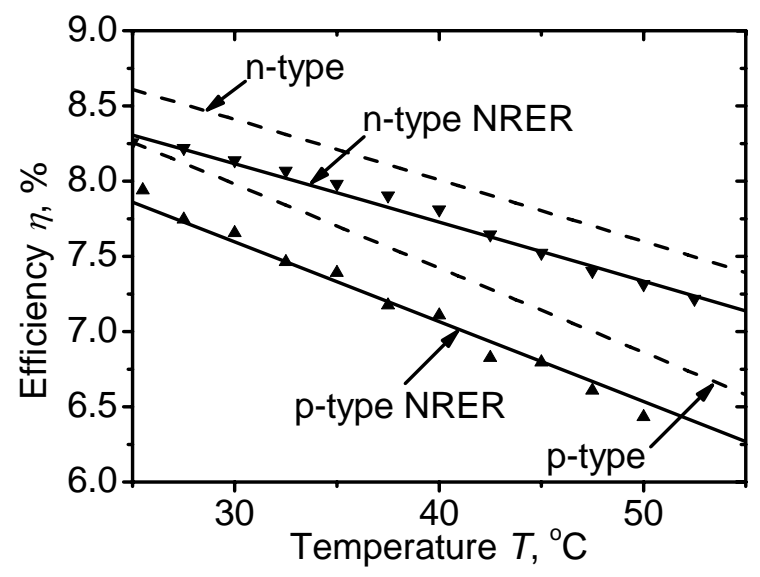

Fig. 6. Theoretical (lines) and experimental (symbols) temperature dependences of photoconversion efficiency. 


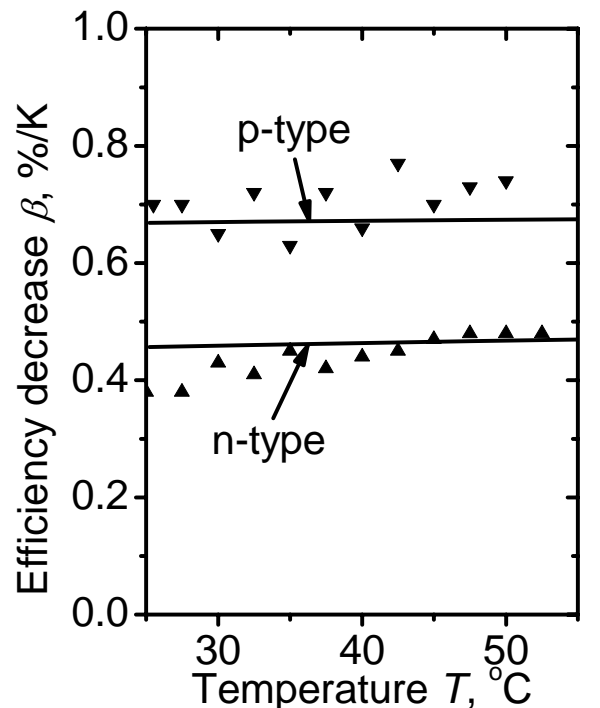

Fig. 7. Theoretical (lines) and experimental (symbols) temperature dependences of photoconversion efficiency temperature coefficient.
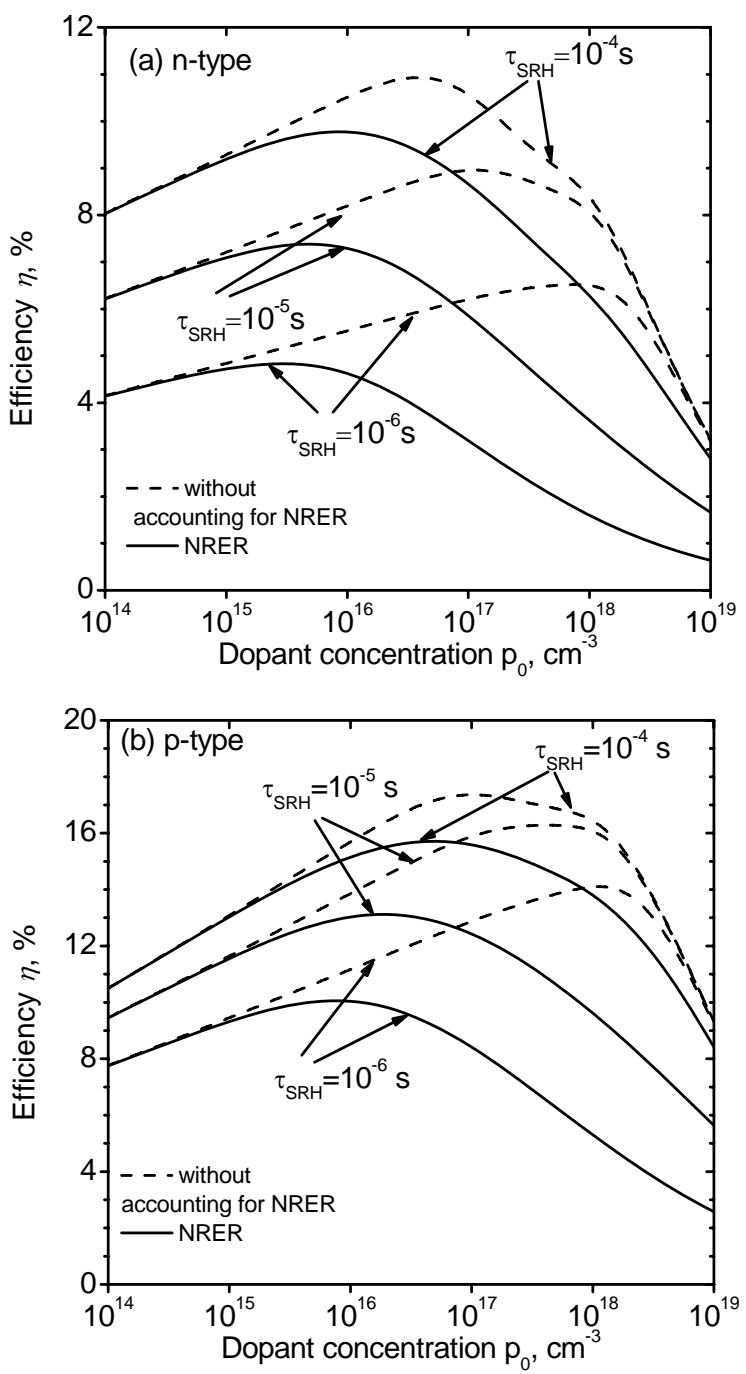

Fig. 8. Theoretical photoconversion efficiency as a function of doping level for (a) $n$-type base and (b) $p$-type base.
Let us now return to the question about the influence of NRER on the SC efficiency with sufficiently low $\tau_{\mathrm{SRH}}$. As follows from Eq. (4), this influence can become apparent, if the condition $n_{0} \geq n_{x}$ is fulfilled. Because for our samples the opposite is true, i.e. $n_{0}<n_{x}$, it can be expected that in this case, the effect of NRER on the efficiency should not be very significant. However, as seen from Fig. 6, the theoretical efficiencies obtained with and without incorporating $\tau_{n r}$ differ quite noticeably, and this difference increases with increasing the doping level.

To validate this observation, we have plotted the theoretical $\eta\left(n_{0}\right)$ curves using all the parameters characterizing our samples except for the ShockleyRead-Hall lifetime, which were set to 100,10 , and $1 \mu$ s, respectively. These curves are shown in Fig. 8 for (a) $n$-type and (b) $p$-type base for AM1.5 irradiation spectrum. As seen in Fig. 8a, the $\eta\left(n_{0}\right)$ curves obtained with and without accounting for NRER are completely different. In particular, as $\tau_{\mathrm{SRH}}$ gets smaller, the maximum of these curves shifts towards lower doping levels, if NRER is taken into account, and it shifts towards larger doping levels, if it is discarded. At the same time, the difference in the efficiency peak values obtained with and without accounting for NRER effect becomes larger. In particular, for $\tau_{\mathrm{SRH}}=1 \mu \mathrm{s}$, this difference can be as high as about $26 \%$ of the efficiency peak value obtained without NRER.

These curves are similar in the case of a $p$-type base, see Fig. 8b. Here, the displacement of the efficiency peaks plotted with NRER taken into account is smaller than in the $n$-type base case. This is caused by the higher surface recombination velocity. The difference between the efficiency values for $\tau_{\mathrm{SRH}}=1 \mu \mathrm{s}$ is about $45 \%$ of the efficiency peak value obtained without NRER.

The curves in Fig. 8 indicate that it is impractical to use high base doping levels in silicon SC with short $\tau_{\mathrm{SRH}}$. Although this fact has been known before from experience, it remained unexplained theoretically.

Last but not least, let us discuss the criteria allowing one to establish the degree of influence of NRER on the photoconversion efficiency of silicon SCs. In order for this recombination mechanism to have no effect on the photoefficiency is that $\tau_{r}<\tau_{n r}$, i.e. the radiative recombination should dominate. Using the expressions for $\tau_{r}$ and $\tau_{n r}$ (see above Eq. (4)), we find that this condition holds for $\tau_{\mathrm{SRH}}>20 \mathrm{~ms}$.

In order for NRER to have a weak effect on the photoconversion efficiency, the inequalities $\tau_{r}>\tau_{n r}$ and $\tau_{n r}>\tau_{\text {Auger }}$ must hold. With the relevant parameter values, we find that the maximum on the $\eta\left(n_{0}\right)$ curves at not too high surface recombination velocities (below $10^{3} \mathrm{~cm} / \mathrm{s}$ ) is at $n_{0} \geq 10^{17} \mathrm{~cm}^{-3}$. The effect of NRER on photoconversion efficiency is weak for $\tau_{\mathrm{SRH}}$ between 0.1 and $20 \mathrm{~ms}$. We note that in this case the maxima of the $\eta\left(n_{0}\right)$ curves plotted with and without taking NRER into account coincide. 
Finally, in the case $\tau_{n r}<\tau_{\mathrm{Auger}}$, i.e. $\tau_{\mathrm{SRH}}<0.1 \mathrm{~ms}$, the effect of NRER on photoconversion efficiency is strong. In this case, the maxima of the $\eta\left(n_{0}\right)$ curves plotted with and without taking this effect into account do not coincide.

\section{Conclusions}

NRER has a strong effect on the photoconversion efficiency in silicon SC under the condition $n_{0}>n_{x}$, in which case the characteristic time of this process becomes shorter than the Shockley-Read-Hall lifetime.

The smaller $\tau_{\mathrm{SRH}}$, the stronger the effect of NRER on photoconversion efficiency. Therefore, at $\tau_{\mathrm{SRH}}$ of the order of $1 \mu \mathrm{s}$, this recombination mechanism is also pronounced when $n_{0}<n_{x}$.

At sufficiently short $\tau_{\mathrm{SRH}}$, NRER is responsible for the shift of the optimal base doping level, at which the photoconversion efficiency has a maximum.

\section{References}

1. Sachenko A.V., Kostylyov V.P., Vlasiuk V.M., Sokolovskyi I.O., and Evstigneev M.A. The influence of the exciton non-radiative recombination in silicon on the photoconversion efficiency. 1. The case of a long Shockley-Read-Hall lifetime. SemiconductorPhysics, Quantum Electronics and Optoelectronics. 2016. 19, No. 4. P. 334-342.
2. Richter A., Glunz S.W., Werner F., Schmidt J., Cuevas A. Improved quantitative description of Auger recombination in crystalline silicon. Phys. Rev. B. 2012. 86. P. 165202.

3. Pikus G.E. Fundamentals of The Theory of Semiconductor Devices. Moscow: Nauka, 1965.

4. Hguyen H.T., Rougieux F.E., Mitchell B., Macdonald D. Temperature dependence of the band-band absorption coefficient in crystalline silicon from photoluminescence. J. Appl. Phys. 2014. 115. P. 043710.

5. Green M.A. Intrinsic concentration, effective densities of states, and effective mass in silicon. $J$. Appl. Phys. 1990. 67, No. 6. P. 2944-2954.

6. Abakumov V.N., Perel V.I., Yassievich I.N. Radiationless Recombination in Semiconductors. St. Petersburg: B.P. Konstantinov St. Petersburg Institute of Nuclear Physics of RAN, 1997 (in Russian).

7. Fahrenbruch A.L., Bube R.H., Fundamentals of Solar Cells: Photovoltaic Solar Energy Conversion. New York: Academic, 1983.

8. Skoplaki E., Palyvos J.A. On the temperature dependence of photovoltaic module electrical performance: A review of efficiency power correlation. Solar Energy. 2009. 83, No. 5. P. 614-624. 